\title{
Extensive Deletion and Sequence Variation of Plasmodium Falciparum Histidine Rich Protein 2/3 (pfhrp2/3) Genes in Ethiopia: Implication for RDT- based Malaria Diagnosis and Control
}

\section{Lemu Golassa ( $\nabla$ lgolassa@gmail.com )}

Addis Ababa University, Aklilu Lemma Institute of Pathobiology https://orcid.org/0000-0002-1216-8711

Alebachew Messele

Addis Ababa University School of Social Work

Alfred Amambua-Ngwa

Medical Research Council

Gote Swedberg

Uppsala Universitet

\section{Research}

Keywords: Plasmodium falciparum, Histidine rich protein 2/3, Deletion, RDTs, Microscopy, Ethiopia

Posted Date: April 15th, 2020

DOl: https://doi.org/10.21203/rs.3.rs-22606/v1

License: (c) (i) This work is licensed under a Creative Commons Attribution 4.0 International License. Read Full License 


\section{Abstract}

Background: Despite remarkable malaria reduction in recent years, malaria remains a public health problem in Ethiopia. With the introduction of rapid diagnostic tests (RDTs), malaria diagnosis has been transformed. However, the Plasmodium falciparum histidine rich protein 2 ( hrp 2) that is targeted by the most widely used RDTs is prone to genetic mutations and gene deletions as observed in recent years. Patients infected with P. falciparum malaria parasites with a deletion in hrp 2/3 gene locus would remain undetected and results in 'false-negatives', which are not treated. Undoubtedly, these untreated infected patients are at risk of developing complicated disease and may further fuel parasite transmission. Hence, molecular targeting of the region across exons and flanking genes has been used to provide greater confirmatory evidence of gene deletions. This study was initiated to determine pfhrp $2 / 3$ gene deletions including the flanking regions.

Methods: A cross-sectional study was conducted to determine the prevalence of hrp 2/3 genes deletion.Finger-prick blood samples were collected from a total of 64 febrile patients attending Adama Malaria Diagnostic Centre in 2015. Thick and thin blood smears were prepared for microscopic slide readings, and parasitaemias were determined. Blood samples were spotted onto filter for parasite DNA extraction.

Results: From a total of 64 microscopically and PCR confirmed P. falciparum infections, 50 were successfully analyzed for deletion of pfhrp2, pfhrp3 and flanking regions. Extensive deletions were observed in the pfhrp2 gene with all 50(100\%) isolates presenting a deletion. This deletion extended downstream towards the Pf3D7 0831900 (MAL7PI.230) gene in 11/50 (22\%) cases. In contrast, only 2/50 (4\%) of samples had deletions for the upstream gene Pf3D7 0831700 (MALPI.228). Similarly, all isolates had deleted the pfhrp3 gene (100\%) and this in $40 \%$ of isolates extended to the downstream flanking region Pf3D7 13272400 (MAL13PI.485) where 40\% samples showed absence of this region. However, the deletion also extended toward the upstream region Pf3D7 081372100 (MAL13PI.475). The deleted pfhrp 3 genomic region also extended upstream to the region Pf3D7 081372100 (MAL13PI.475) with 49/50 (95\%) of the isolates exhibiting absence of the locus.

Conclusion: As patient recruitment was not done on pfhrp 2/3-based RDTs, it is impossible to know if the isolates would test negative or positive in the absence of hrp 2/3 genes. Indeed, the sequence variation and high frequencies of deletion in pfhrp2 and pfhrp3 genes in Ethiopian isolates most likely will have a negative influence on the performance of currently used pfhrp2 RDTs. This study confirms the presence of that P. falciparum parasite population with extensive deletions of the pfhrp 2 and pfhrp3 genes in Ethiopia and this calls for a countrywide surveillance to determine the extent of these deletions and its effect on routine malaria diagnosis. Keywords: Plasmodium falciparum , Histidine rich protein 2/3, Deletion, RDTs, Microscopy, Ethiopia

\section{Introduction}


In Ethiopia, Plasmodium falciparum and Plasmodium vivax co-exist with relative proportions of 0.60 and 0.40 of malaria cases, respectively $[1,2]$. Nearly $68 \%$ of the landmass, where $60 \%$ of the population lives, is favourable for malaria transmission [1, 3]. Depending on the local malaria transmission intensity or endemicity class, the epidemiology of malaria varies geographically [4]. Like in many most of Africa, the incidence of malaria has been substantially reduced in Ethiopia which also reported a substantial $40 \%$ reduction in incidence by the year 2020 [5]. Ethiopia is on track to achieve the 2020 milestone by reducing the incidence of malaria by $40 \%$ and also aligns with the World Health Organization (WHO) Global Technical Strategy (GTS) by intensifying existing malaria control activities to eliminate malaria by 2030 [5]. Much of this can be accredited to the national malaria control and prevention strategies, which have introduced insecticide treated nets, insect residual spray and a change in drug policy. Essentially, rapid diagnostic tests (RDTs) have been introduced to improve early diagnosis of malaria in remote areas where microscopic examination of blood smears seems impractical.

RDTs are immune-chromatographic tests that are designed to detect malaria specific antigens such as lactate dehydrogenase or aldolase for pan-malaria diagnosis and pfhrp2 for $P$. falciparum specific diagnosis [6]. Since the first report detailing the deletion of pfhrp2 in Peru and its impending consequences was published in 2010 , several studies have shown the global spread of parasites lacking pfhrp2 gene and its flanking regions [7]. In Eritrea, $P$. falciparum lacking histidine-rich protein has become a major threat to malaria control programs $[8,9]$. As they are not detected by pfhrp2/3-based RDTs and remain untreated, parasites carrying the $p f h r p 2 / 3$ gene deletions have a fitness advantage posing a challenge to progress made in malaria control and elimination.

Particularly in South America, Peru has shown a high prevalence of these parasites with $41 \%$ deletion in pfhrp2 and 70\% deletion in pfhrp3 gene, this was also confirmed by another study which showed an increase in prevalence from 20.7 to $40.6 \%$ within two years, in another region of the country [10]. Similarly, a study on P. falciparum outbreak on the northern Pacific coast of Peru (2010-2012) using 54 samples showed all $100 \%$ samples lacked the pfhrp2 gene [11]. Following this, several countries have reported occurrence of pfhrp2 and pfhrp3 gene deletions [9, 12-21]. For highly malaria endemic Africa, the prevalence of the deletion from recent report have generally been low or absent. However, very high prevalence has been reported for Eritrea, which shares a border with Ethiopia. So far, no studies have been conducted in Ethiopia to survey or detect the status of pfhrp2 deletions in the country. Undoubtedly, parasites with deleted $h r p 2 / 3$ genes will be a significant setback for malaria elimination in this region since RDT-based diagnostics are a key pillar in effective malaria management and control. Following the WHO recommendation for pfhrp2/3 surveys and surveillance activities to target neighbouring countries where deletions have been reported, this study investigates the extent of pfhrp2/3 deleted $P$. falciparum parasites in Ethiopia. Molecular targeting the region across exons and flanking genes would provide greater confirmatory evidence of gene deletions.

\section{Methods}

\section{Sample collection and diagnosis of malaria}


Finger-prick blood samples were collected from a total of 64 febrile patients attending Adama Malaria Diagnostic Centre in 2015. Thick and thin blood smears were prepared for microscopic slide readings, and parasitaemias were determined. Blood samples were spotted onto filter for parasite DNA extraction.

\section{Parasite Dna Extraction, Pcr Analysis And Dna Sequencing}

Parasite DNA was extracted from blood spots on filter paper using chelex extraction methods as described elsewhere [22].

\section{Detection and amplification of hrp2/3 genes}

Successful amplification of pfhrp2 and pfhrp3 was made for a total of 50 samples confirmed both by microscope. Exon 2 of both genes was amplified to demonstrate the presence or absence of the pfhrp2 and pfhrp3 genes and their flanking regions using published protocols and primers (Additional file 3). Briefly, pfhrp3 gene (475 to 485) was amplified using the following PCR conditions: denaturing at $95^{\circ} \mathrm{C}$ for 3 minutes followed by $94^{\circ} \mathrm{C}$ for 15 seconds ( 36 cycles), annealing at $55^{\circ} \mathrm{C}$ for 15 seconds ( 35 cycles), elongation $60^{\circ} \mathrm{C}$ for $1: 30$ minutes $\left(36\right.$ cycles) and cooling at $16^{\circ} \mathrm{C}$. Amplification of the pfhrp 2 genes (475 to 485) was made as shown in Table 1. 
Table 1

Outer and nested PCR conditions used to amplify

pfhrp2 gene.

\begin{tabular}{|llll|}
\hline Hrp2-Outer & Temp & Time & Process \\
\hline Step 1 & 95 & $3 \mathrm{~min}$ & Denaturation \\
\hline Step 2 & 94 & $15 \mathrm{sec}$ & \\
Step 3 & 55 & $15 \mathrm{sec}$ & Annealing \\
\hline Step 4 & 60 & $1: 30 \mathrm{~min}$ & Elongation \\
\hline Step 5 & 72 & $5 \mathrm{~min}$ & \\
\hline Step 6 & 16 & $\infty$ & Cooling \\
\hline Hrp2-Nested & Temp & Time & Process \\
\hline Step 1 & 95 & $3 \mathrm{~min}$ & Denaturation \\
\hline Step 2 & 94 & $15 \mathrm{sec}$ & \\
\hline Step 3 & 55 & $15 \mathrm{sec}$ & Annealing \\
\hline Step 4 & 60 & $1 \mathrm{~min}$ & Elongation \\
\hline Step 5 & 72 & $5 \mathrm{~min}$ & \\
\hline Step 6 & 16 & $\infty$ & Cooling \\
\hline
\end{tabular}

For amplification of pfhrp2, two different primer sets were used. The first one targets the repeat region which harbours most of the variable part of the gene (Additional file 3). The second primer set targets an intron region and for this primer set 5 samples gave adequate PCR products and their sequences were determined. Successful PCR products were purified by the GeneJet PCR Cleanup Kit from Thermo Fisher Scientific and sent for sequence determination at Eurofins genomics, Germany. Sequences were analysed by the 4peaks program (A. Griekspoor and Tom Groothuis, nucleobytes.com).

\section{Ethical Issues}

The study was approved by Aklilu Lemma Institute of Pathobiolohy, Addis Ababa University, Institutional review Board. Written consent and/or assent were obtained from each study participants.

\section{Results}

\section{PCR confirmation of P. falciparum infections}


Febrile patients travelled from various areas to Adama Malaria Diagnostic Centre for malaria diagnosis. The male: female ratio was 3.1:1. Participant's mean age was 25.2 years (range 11-48). A total of 50 specimens met the inclusion criteria for this study by yielding positive results for amplification for both 18 S rRNA gene. Using 18 S rDNA PCR, all 50 specimens were positive for $P$. falciparum. Additionally, all samples were amplified for the pfmdr 1 gene. The minimum parasite density reported by microscopy was 400 parasites $/ \mu \mathrm{L}$, which would indicate that this is the threshold for detection by the field microscopists.

\section{Genetic deletion of pfhrp2 and pfhrp3 and their flanking genes}

From the 64 microscopically and PCR confirmed $P$. falciparum infections, 50 were successfully analysed for pfhrp2, pfhrp3 and flanking regions described above (Table 2). Extensive deletion was observed in the pfhrp2 gene with all $50(100 \%)$ isolates presenting a deletion. This deletion extended downstream towards the Pf3D7 0831900 (MAL7PI.230) gene in 11/50 (22\%) of cases. In contrast only 2/50 (4\%) of samples had deletions for the upstream gene Pf3D7 0831700 (MALPI.228).

The samples contained different AT repeat sequences (one sample with 10 repeats, and four samples with 17 repeats) (data not shown) suggesting that the deletion in this region did not involve the entire region. Indeed, the positive PCR products were from a small part covering an intron region where these repeats are found (Additional file 3).

The results for pfhrp3 and flanking regions were comparable. Here, only the repeat region was analysed and all isolates had deletions in the pfhrp3 gene (100\%) and like pfhrp2 this deletion extended towards the downstream flanking region Pf3D7 13272400 (MAL13PI.485) where 40\% samples shown absence of this region. However, the extension of the deletion was more prevalent towards the upstream region Pf3D7 081372100 (MAL13PI.475), where 49/50 (95\%) of the isolates exhibited absence of the loci. The extension of deletions in pfhrp2 and pfhrp3 genes can be viewed from the Additional files 1 and 2.

By targeting six regions in the $h r p 2 / 3$ and flanking regions, different deletion patterns were observed in Ethiopian $P$. falciparum clinical samples (Table 2). A greater proportion of parasite isolates had deleted the gene located 3' of pfhrp2, PF3D7_0831900, compared to the flanking gene 5', PF3D7_0831700. In sharp contrast, the 5' flanking gene PF3D7_1372100 (upstream of pfhrp3) showed more deletions than the 3' flanking region PF3D7_1372400 region (downstream of pfhrp3). As such the most common pattern exhibited in the isolates was the presence of the the two flanking regions for pfhrp 2 in combination with the downstream flanking region for $h r p 3$. This was followed by isolates that had deleted the downstream flanking region for $h r p 3$ but retained the two flanking for $h r p 2$. Notably, only one isolate showed the presence for all four flanking regions. 
Table 2

Results of PCR amplification of pfhrp2, pfhrp3 and their respective flanking genes in $P$. falciparum samples collected in Ethiopia.

\begin{tabular}{|lllllll|}
\hline No. & PF3D7_0831700 & HRP2 & PF3D7_0831900 & PF3D7_1372100 & HRP3 & PF3D7_1372400 \\
\hline 20 & + & - & + & - & - & + \\
\hline 16 & + & - & + & - & - & - \\
\hline 8 & + & - & - & - & - & + \\
\hline 3 & + & - & - & - & - & - \\
\hline 2 & - & - & + & - & - \\
\hline 1 & + & - & + & + & + \\
\hline 50 & & & & & + \\
\hline $\begin{array}{l}\text { The association between parasite density and } h r p 2 \text { deletion was not evaluated due to the absence of } \\
\text { hrp2/hrp3 positive samples. }\end{array}$ \\
\hline
\end{tabular}

\section{Discussion}

RDTs have become extremely essential for implementing early diagnosis and prompt effective treatment to substantially reduce the incidence rate of malaria in Africa. Nowadays, RDTs are the most widely used malaria diagnostics especially in areas where microscopic diagnosis is impractical for several reasons. However, variation in the performance of RDTs variation has been chronicled, probably driven by known deletion polymorphism targeting pfhr2/hrp3 loci. However, the prevalence and dynamics of pfhrp2 deleted strains has not been extensively investigated, especially in Africa. This study is one of the first reports showing an extensive deletion of $p f h r p 2 / 3$ genes in clinical isolates from Ethiopia. This was evident for pfhrp2 and its structural homolog pfhrp3 as well as their respective flanking genes. In order to rule out the possibility that this was caused by primers we used alternative primers with different binding sites and amplification conditions. In addition, we used another locus, pfmdr 1 , to rule out the possibility of low DNA quality being an issue. Furthermore, we used 18ssRNA to confirm microscopically determined $P$. falciparum positive specimens.

From all the six targets of pfhrp $2 / 3$ and flanking regions (Table 2), at least one locus was amplified for the 50 studied samples from a total of 64 confirmed $P$. falciparum clinical samples. In all 50 samples, pfhrp $2 / 3$ genes had deletions which continued to their flanking regions although deletions here were to a lesser degree. The lack of prior data on pfhrp $2 / 3$ deleted parasites in the country limits our investigation on whether these deletions are recent events or were present prior to the introduction of pfhrp2-based RDTs. For Peru, for instance, the appearance of these parasites was evident before the introduction of RDTs, which perhaps show the strength of selective forces on these deletions [7]. In contrast, a recent 
mathematical modelling study showed that the use of $p$ fhrp2-based RDTs is sufficient to select $P$. falciparum parasites lacking this protein.

Interestingly, in Ethiopia pfhrp2-based RDTs are far more popular than pLDH-based RDTs which could be because of their higher sensitivity for $P$. falciparum diagnosis. However, the fact that both pfhrp2 and pfhrp3 deletions were detected in all of our samples probably indicate that this could have been a result of a recent selection. Furthermore, parasites lacking both genes are quite rare to find in other African countries, though this is quite prevalent in South American countries [7, 10]. Indeed, a recent whole genome study on $P$. falciparum isolates from 15 African countries has shown a highly divergent Ethiopian $P$. falciparum population, defining a genomic background that could likely dictate a different response to selective forces on the parasite [23]. A similar neighbouring parasite population in Eritrea also reported very high frequencies of these deletions $[8,9]$.

RDT-based treatment could be a determining factor to selectively clear non-deleted infections and hence increase the rate of spread of parasites with deletions [24]. However, the benefits of this mutation to the parasites and if RDT-guided treatments may have selected for pfhrp2-deleted mutants are yet to be determined. In Eritrea where malaria prevalence is quite similar to Ethiopia, pfhrp2-negative parasites had lower genetic diversity compared with that of pfhrp2-positive parasites and formed a closely related cluster probably caused by selection by use of pfhrp2-based RDTs [7]. Given that the observation of partial or complete deletion of the pfhrp2 gene in 2010 in South America sparkled the recommendation against the use of $p$ fhrp2-based RDTs in these areas, the results here are relevant for future policy in Ethiopia [25-27]. This needs to take in consideration some limitations, such as the absence of data on diagnostics outcome for the samples based on RDTs that targets the pfhrp2/3 genes. The fact that patient recruitment was not made using pfhrp2/3-based RDTs while the samples were collected makes it difficult to determine if the isolates would test negative and/or positive in the absence of $p f h r p 2 / 3$ genes. Further studies using microscopy and pfhrp2/3-based RDTs are required at large scale to determine the extent of $p f h r p 2 / 3$ gene deletions and the role the deletion could play in the test results in Ethiopia. The samples were also collected from one region (health centre) of the country, warranting future and extensive studies to further inform the national malaria control program (NMCP) on diagnostic approaches in Ethiopia as the elimination agenda in pursued. The routine use of RDTs may allow the parasites to escape detection by phrp2-based RDTs and may be selected to expand in the population. Worth noting is that although the pfhrp2/3 genes were deleted and/or absent, the respective flanking regions were amplified and they may contribute to reactivity of pfhrp2/3-based RDTs. Indeed, the sequence variation and deletion in $P f h r p 2 / 3$ genes in Ethiopian isolates may not likely to negatively influence performance of currently used pfhrp2 RDTs given that the RDTs targeting hrp2/3 genes are massively used in the area.

\section{Conclusions}

P. falciparum parasite populations with deletions of the pfhrp2 and pfhrp3 genes are present in Ethiopia and further large scale studies are required to identify the prevalence of deletion and its effect on current 
RDTs-based diagnosis, towards adaptation of malaria control strategies.

\section{Abbreviations}

DNA

Deoxyribonucleic acid

GTS

Global Technical Strategy

Hrp2/3

histidine rich protein $2 / 3$

NMCP

National malaria control program

Pfhrp $2 / 3$

Plasmodium falciparum histidine rich protein 2

PCR

Polymerase chain reaction

pLDH

Plasmodium lactate dehydrogenase

RDTs

rapid diagnostic tests

RNA

Ribonucleic acid

rRNA

18S ribosomal RNA

rDNA

$18 \mathrm{~S}$ ribosomal DNA

WHO

World Health Organization

\section{Declarations}

\section{Authors' contributions}

LG collected the samples and drafted the manuscript. AM and AAN, analysed and interpreted the data. GS did the molecular test. All authors discussed the results and contributed to the final manuscript. All authors read and approved the final manuscript

Author details

${ }^{1}$ Aklilu Lemma Institute of Pathobiology, Addis Ababa University, ${ }^{2} \mathrm{MRC}$ Unit The Gambia at the London School of Hygiene and Tropical Medicine, ${ }^{3}$ Department of Medical Biochemistry and Microbiology, Uppsala University, Sweden 
Competing interests

The authors declare that they have no competing interests

Funding

Addis Ababa University, Aklilu Lemma Institute of Pathobiology, Thematic Research.

Acknowledgements

This work was in part supported through the DELTAS Africa Initiative [DELGEME grant 107740/Z/15/Z]. Laboratory technicians are dully acknowledged for their great efforts in sample collection and malaria diagnosis.

\section{Availability of data and materials}

All data and materials supporting the results of the manuscript will be available at gote.swedberg@imbim.uu.se and Igolassa@gmail.com upon request

\section{Ethics approval and consent to participate}

Ethical approval for the study was granted by Aklilu Lemma Institute of Pathobiology, Addis Ababa University Institutional Review Board.

\section{References}

1. World Health Organization (WHO). World malaria report 2015. Geneva: World Health Organization; 2015.

2. Taffese HS, Hemming-Schroeder E, Koepfli C, Tesfaye G, Lee M-c, Kazura J, et al. Malaria epidemiology and interventions in Ethiopia from 2001 to 2016. Infectious Diseases of Poverty. 2018;7:103.

3. Ethiopian Federal Ministry of Health EFMH). An epidemiological profile of malaria in Ethiopia. Addis Ababa: Public Health Institute, Ministry of Health; 2014.

4. Snow RW, Marsh K. The consequences of reducing transmission of Plasmodium falciparum in Africa. Adv Parasitol. 2002;52:235-64.

5. Federal Democratic Republic of Ethiopia Ministry of Health (FDREMH). National Malaria Elimination Roadmap. Addis Ababa. 2016. p. 1-32.

6. Murray CK, Bennett JW. Rapid diagnosis of malaria. Interdiscip Perspect Infect Dis. 2009;415953.

7. Gamboa D, Ho MF, Bendezu J, Torres K, Chiodini PL, Barnwell JW, Incardona S, Perkins M, Bell D, McCarthy J, Cheng Q. A large proportion of P. falciparum isolates in the Amazon region of Peru lack pfhrp2 and pfhrp3: implications for malaria rapid diagnostic tests. PLoS One. 2010;5(1):e8091.

8. Berhane A, Russom M, Bahta I, Hagos F, Ghirmai M, Uqubay S. Rapid diagnostic tests failing to detect Plasmodium falciparum infections in Eritrea: an investigation of reported false negative RDT results. Malar J. 2017;16:105.

9. Berhane A, Anderson K, Mihreteab S, Gresty K, Rogier E, Mohamed S, et al. Major threat to malaria control programs by Plasmodium falciparum lacking histidine-rich protein 2, Eritrea. Emerg Infect Dis. 
2018;24:462-70.

10. Maltha J, Gamboa D, Bendezu J, Sanchez L, Cnops L, et al. Rapid Diagnostic Tests for Malaria Diagnosis in the Peruvian Amazon: Impact of pfhrp2 Gene Deletions and Cross-Reactions. PLoS One. 2012;7:e43094.

11. Houze S, Boly MD, Le Bras J, Deloron P, Faucher JF. pfhrp2 and PfLDH antigen detection for monitoring the efficacy of artemisinin-based combination therapy (ACT) in the treatment of uncomplicated falciparum malaria. Malar J. 2009;8:211.

12. Kozycki CT, Umulisa N, Rulisa S, Mwikarago El, Musabyimana JP, Habimana JP, et al. False-negative malaria rapid diagnostic tests in Rwanda: impact of Plasmodium falciparum isolates lacking hrp2 and declining malaria transmission. Malar J. 2017;16:123.

13. Koita OA, Doumbo OK, Ouattara A, Tall LK, Konare A, Diakite M, et al. False-negative rapid diagnostic tests for malaria and deletion of the histidine-rich repeat region of the hrp2 gene. Am J Trop Med Hyg. 2012;86:194-8.

14. Menegon M, L'Episcopia M, Nurahmed AM, Talha AA, Nour BYM, Severini C. Identifcation of Plasmodium falciparum isolates lacking histidine-rich protein 2 and 3 in Eritrea. Infect Genet Evol. 2017;55:131-4.

15. Gupta H, Matambisso G, Galatas B, Cistero P, Nhamussua L, Simone W, et al. Molecular surveillance of pfhrp2 and pfhrp3 deletions in Plasmodium falciparum isolates from Mozambique. Malar $\mathrm{J}$. 2017; 16:416.

16. Funwei R, Nderu D, Nguetse CN, Thomas BN, Falade CO, Velavan TP, et al. Molecular surveillance of pfhrp2 and pfhrp3 genes deletion in Plasmodium falciparum isolates and the implications for rapid diagnostic tests in Nigeria. Acta Trop. 2019;196:121-5.

17. Wurtz N, Fall B, Bui K, Pascual A, Fall M, Camara C, et al. Pfhrp2 and pfhrp3 polymorphisms in Plasmodium falciparum isolates from Dakar, Senegal: impact on rapid malaria diagnostic tests. Malar J. 2013;12:34.

18. Parr JB, Vity R, Doctor SM, Janko M, Carey-Ewend K, et al. Pfhrp2-deleted Plasmodium falciparum parasites in the Democratic Republic of the Congo: a national cross-sectional survey. J Infect Dis. 2017;216:36-44.

19. Nderu D, Kimani F, Thiong'o K, Akinyi M, Karanja E, Meyer CG, et al. pfhrp2-pfhrp3 diversity among Kenyan isolates and comparative evaluation of $p f h r p 2 / p L D H$ malaria RDT with microscopy and nested PCR methodologies. Parasitol Int. 2018;67:793-9.

20. Amoah LE, Abankwa J, Oppong A. Plasmodium falciparum histidine rich protein-2 diversity and the implications for pfhrp 2- based malaria rapid diagnostic tests in Ghana. Malar J. 2016;15:101.

21. Beshir KB, Sepulveda N, Bharmal J, Robinson A, Mwanguzi J, Busula AO, et al. Plasmodium falciparum parasites with histidine-rich protein 2 ( $p$ fhrp2) and pfhrp3 gene deletions in two endemic regions of Kenya. Sci Rep. 2017;7:14718.

22. Plowe $\mathrm{C}$, Djimde A, Bouare $\mathrm{M}$, Doumbo $\mathrm{O}$, Wellems TE. Pyrimethamine and proguanil resistanceconferring mutations in Plasmodium falciparum dihydrofolate reductase: polymerase chain reaction 
methods for surveillance in Africa. Am J Trop Med Hyg. 1995;52:565-8.

23. Amambua-Ngwa A, Amenga-Etego L, Kamau E, Amato R, Ghansah A, Golassa, et al. Major subpopulations of Plasmodium falciparum in sub-Saharan Africa. 2019;365: (6455) 813-816.

24. Watson Oliver, Slater HC, Verity R, Parr JB, Mwandagalirwa MK, Tshefu A, Meshnick SR, Ghani AC. Modelling the drivers of the spread of Plasmodium falciparum hrp2 gene deletions in sub-Saharan Africa. eLife. 2017;2:6: e25008.

25. Akinyi S, Hayden T, Gamboa D, Torres K, Bendezu J, Abdallah JF, Griffing SM, Quezada WM, Arrospide N, De Oliveira AM, Lucas C, Magill AJ, Bacon DJ, Barnwell JW, Udhayakumar V. Multiple genetic origins of histidine-rich protein 2 gene deletion in Plasmodium falciparum parasites from Peru. Sci Rep. 2013;3:1-8.

26. Abdallah JF, Okoth S, Fontecha GA, Torres R, Banegas El, Matute M, Bucheli S, Goldman IF, de Oliveira A, Barnwell JW, Udhayakumar V. Prevalence of pfhrp2 and pfhrp3 gene deletions in Puerto Lempira. Honduras Malar J. 2015;14:19.

27. Cheng Q, Gatton ML, Barnwell J, Chiodini P, McCarthy J, Bell D, Cunningham J. Plasmodium falciparum parasites lacking histidine-rich protein 2 and 3: a review and recommendations for accurate reporting. Malar J. 2014;13:283.

28. Additional file 3. Primer sequences for pfhrp2, pfhrp3 and their respective flanking genes.

\section{Supplementary Files}

This is a list of supplementary files associated with this preprint. Click to download.

- Additionalfile1.doc

- Additionalfile3.doc

- Additionalfile2.doc 